\title{
Clinical characteristics and outcomes of patients with overlapping Miller Fisher syndrome and myasthenia gravis
}

Jun-Liang Yuan ${ }^{1}$, Yan Xing ${ }^{2}$, Wen-Li Hu ${ }^{1}$

\author{
'Department of Neurology, Beijing Chaoyang Hospital, Capital Medical University, \\ Chaoyang District, Beijing, China \\ 2Department of Neurology, Aviation General Hospital, China Medical University \\ and Beijing Institute of Translational Medicine, Chinese Academy of Sciences, \\ Chaoyang District, Beijing, China
}

Submitted: 30 May 2017

Accepted: 31 July 2017

Arch Med Sci 2020; 16 (1): 233-236

DOI: https://doi.org/10.5114/aoms.2020.91294

Copyright @ 2019 Termedia \& Banach

Myasthenia gravis (MG) is an autoimmune disease characterized by muscle weakness and fatigue, which is caused by autoantibodies directed against acetylcholine receptors (AChR) at the neuromuscular junction [1]. The prognosis is relatively favorable with optimum symptomatic, immunosuppressive and supportive treatment. Pyridostigmine is the preferred symptomatic treatment, and corticosteroids, azathioprine and thymectomy are first-line immunosuppressive therapies [2].

Guillain-Barré syndrome (GBS) is the most common and most severe acute paralytic neuropathy, with about 100,000 people developing the disorder every year worldwide [3]. Under the terms of GBS are several recognizable variants with distinct clinical and pathological features. As a rare variant of GBS, Miller Fisher syndrome (MFS) is an immune-mediated neuropathy that involves the triad of symptoms of acute ophthalmoplegia, ataxia and areflexia, also with positive GQ1b antibody. The current available treatments include intravenous immunoglobulin (IVIG), plasmapheresis, and supportive care including treatment of underlying infections and physical therapy [4]. MFS usually runs a benign clinical course, with case fatality of $<5 \%$ [5].

Up to now, the occurrence of MG and GBS overlapping in the same patient is quite scarce. To our best of knowledge, only four cases have previously been reported regarding the temporal coincidence between MG and MFS [6-9]. Here, we review all the above 4 cases, and we also describe a new case of our own. We also aimed to summarize the clinical characteristics and to elucidate the underlying mechanisms in such a rare overlapping syndrome.

Literature was reviewed through the databases of PubMed, Embase, Cochrane Library and Science Direct from January 1982 to June 2017, and the articles were restricted to those published in English. Key search terms included "Guillain-Barré syndrome", "Miller Fisher syndrome" and "myasthenia gravis". Patients with combined MG and MFS were identified and their clinical data such as gender, age, nationality, past history, precipitating factors, clinical presentations, laboratory examinations, cerebrospinal fluid (CSF) findings, AChR antibody, anti-GQ1b antibody, thymoma, treatment and prognosis during follow-up were all investigated in detail.

\author{
Corresponding author: \\ Wen-Li Hu \\ Department \\ of Neurology \\ Beijing Chaoyang Hospital \\ Capital Medical University \\ 8 Gongti South Road \\ Chaoyang District \\ Beijing 100020, China \\ Phone: +861085231398 \\ E-mail: yuan_doctor@ \\ outlook.com
}


We herein present a case with a temporal coincidence between MG and MFS. A 72-year-old man first presented with acute bilateral ptosis, ophthalmoplegia, diplopia and dysphagia for one week. He was healthy except with a smoking history of 30 years. No precipitating upper respiratory or gastrointestinal infective symptoms were found. Neurological examination revealed bilateral ptosis, ophthalmoplegia, bulbar palsy, slight weakness of limbs, areflexia, limb ataxia, and no response of plantar flexor reflexes. Magnetic resonance imaging (MRI) of the patient's brain and spinal cord yielded normal findings. The serum biomarkers of tumor and paraneoplastic syndrome were normal. The CSF showed an intracranial pressure of $90 \mathrm{~mm} \mathrm{H}_{2} \mathrm{O}$ (reference range: 80-180), with protein $88 \mathrm{mg} / \mathrm{dl}$ (reference range: 20-40) and leukocytes 4 cells $/ \mathrm{mm}^{3}$ (reference range: $0-8$ ). High-resolution computed tomography (CT) of the chest revealed a huge $8 \mathrm{~cm} \times 3.3 \mathrm{~cm}$ thymoma (Figure 1). The neurophysiological tests were performed after 10 days of his admission. Nerve conduction test (NCT) showed mild extended latency of bilateral median nerve, and conduction velocity reduction in the bilateral median nerve; however, the nerve conduction results (tibial and sural nerve) of the lower extremities were within normal limits. The repetitive nerve stimulation (RNS) test indicated decremental responses both at $2-5 \mathrm{~Hz}$ and $10-30 \mathrm{~Hz}$ stimulation. The prostigmine test was positive, with the partial improvement of the bilateral ptosis and external ophthalmoplegia. Positive antiAChR and anti-GQ1b antibody were observed from blood serum; however, the other antibodies were negative. The patient underwent oral pyridostigmine (60 mg three times one day) and IVIG treatment for 5 days. However, these two strategies did not improve the patient's symptoms prominently. About 3 months later, this patient underwent thymectomy at the department of thoracic surgery in our hospital. Thymoma was also confirmed by histopathological examination. After the thymectomy, he was also treated with

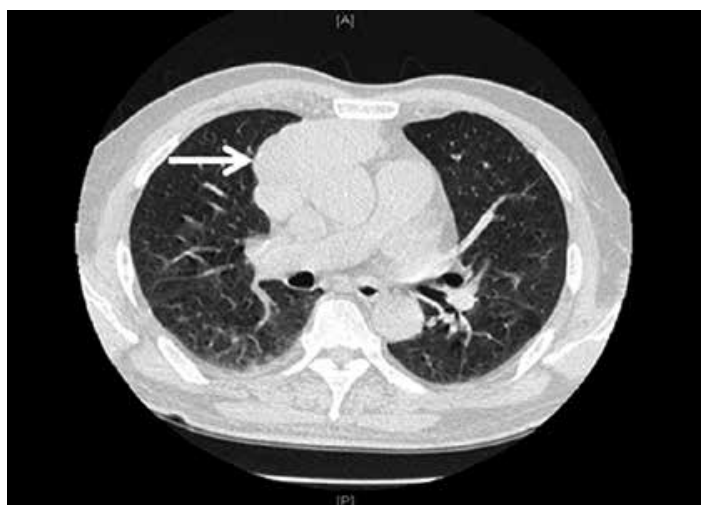

Figure 1. High-resolution CT of chest revealed a big $8 \mathrm{~cm} \times 3.3 \mathrm{~cm}$ thymoma pyridostigmine (60 $\mathrm{mg}$ three times in 1 day) and he recovered fully with a good prognosis in the subsequent 6 months of follow-up.

Of 5 cases, 2 cases had preceding factors. Elevated CSF protein level without pleocytosis (albumino-cytologic dissociation) was found in 3 cases, there were 3 cases with reduced NCT, 4 cases with positive RNS, 4 cases with positive anti-AChR antibodies, 5 cases with positive anti-GQ1b antibody, and 1 case with coexisting with thymoma. The treatment included pyridostigmine in 3 cases, prednisolone in 1 case, intravenous immunoglobulin in 3 cases, and plasmapheresis in 1 case. The functional outcome was favorable according to the adopted scale by Hughes (0-1) (Table I).

As for our case, our patient presented with acute bilateral ptosis, diplopia, ophthalmoplegia, ataxic gait, bulbar dysfunction, weakness of limbs and areflexia. MG was diagnosed according to the clinical features, electrophysiological data, positive anti-AChR antibody and radiological findings of thymoma. The diagnosis of MFS was established based on the classic triad of ataxia, areflexia, and ophthalmoplegia, albumino-cytologic dissociation in CSF, and also confirmed by positive anti-GQ1b antibody. As a result, according to the clinical characteristics, electrophysiological findings, laboratory data, the improvement of anti-acetylcholinesterase and IVIG, especially the effect of thymectomy, the diagnosis of the coincidence between MG and MFS was therefore established.

MG and MFS are well-described autoimmune disorders, which are accepted to be heterogeneous with autoantibodies directed against the neuromuscular junction and peripheral nerve with several different antigens, respectively. However, the exact causes of MG or MFS have remained unclear. The annual incidence of MG ranges from 3 to 30 per 1,000,000 [10]. The annual incidence of MFS is around one patient per one million population [5]. Furthermore, the combination of $M G$ and MFS is extremely rare. Although MG and MFS may have some clinically similar symptoms, the differential diagnosis should be made based on the typical clinical characteristics, and different neurophysiological findings. The serological confirmation with the anti-GQ1b antibody is available and allows for greater diagnostic certainty in the face of confounding symptoms.

Autoimmunity response may play a vital role in understanding the pathophysiological mechanism in both MG and MFS. MFS has been reported to arise through molecular mimicry with microbial oligosaccharides [11]. The theory of molecular mimicry postulates that infectious agents and self-antigen initiate the process of MG and MFS concurrently. It has also been speculated that pathogenic activation of the immune system may 


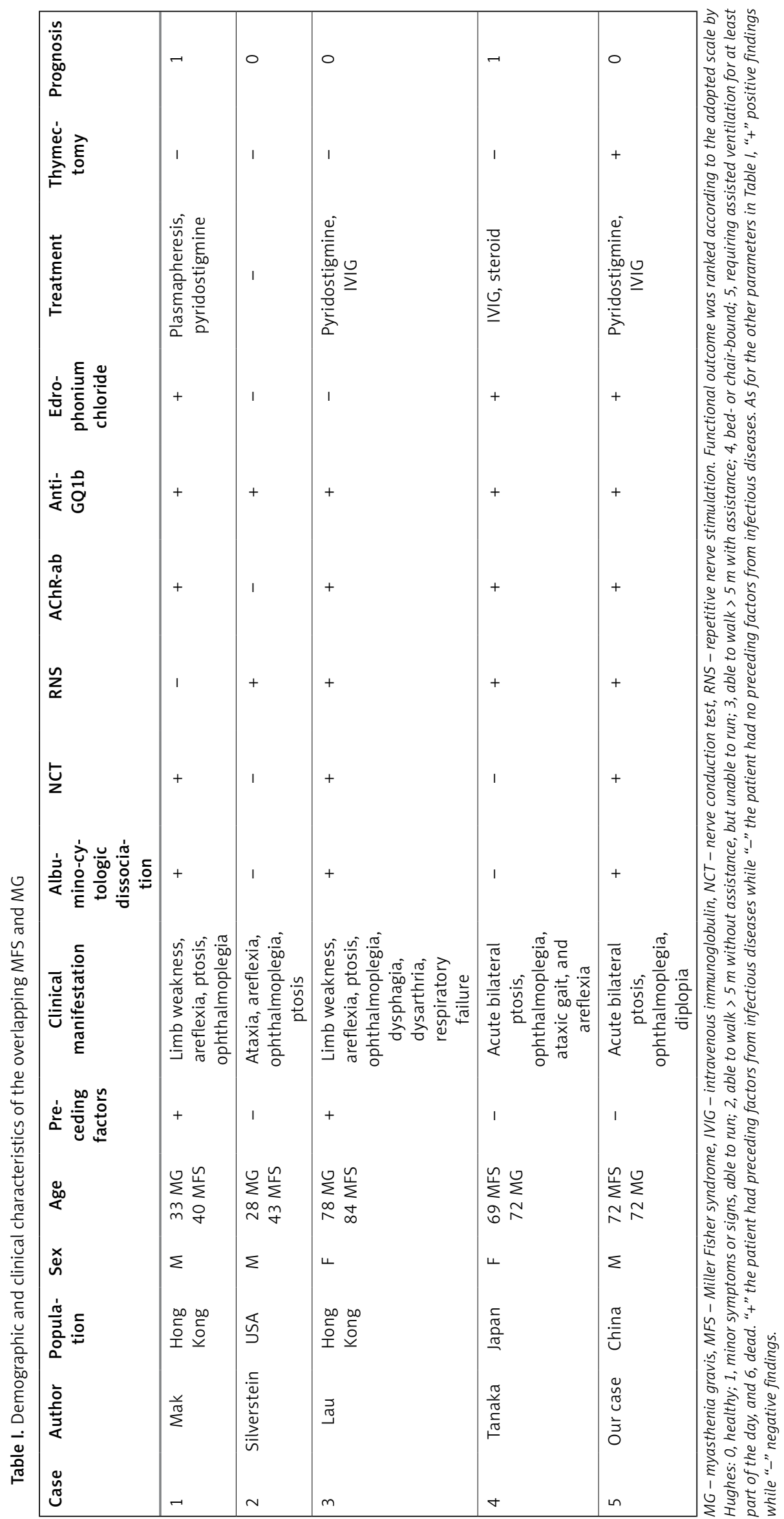


lead to the production of cross-reacting antibodies against peripheral nerve and AChR at neuromuscular junctions from the evidence of the experimental findings [12]. Additionally, anti-GQ1b antibodies lead to impaired neuromuscular transmission in a mouse diaphragm model [13]. Another in-vitro study using mouse hemidiaphragm also demonstrated that the neuromuscular junction is an antigenic target and primary site of pathology underlying in MFS [11]. Furthermore, some clinical evidence also supports the presence of molecular mimicry between gangliosides and antecedent infectious agents in patients with GBS.

Another hypothesis proposed is that thymoma or thymus hyperplasia-associated multiorgan autoimmunity may also play an important role in the process of autoimmunity. It has been reported that approximately $8-15 \%$ of MG are complicated by autoimmune diseases such as immune thyroid disease and systemic lupus erythematosus, which has been well described [14]. The association of MG or MFS with some other autoimmune diseases such as autoimmune thyroiditis has also been previously described [15-17]. In our study, only one patient suffered from thymoma; however, the mass of thymoma. may be considered to be a commonly involved immune organ of MG.

In conclusion, we herein described a case involving the overlap of MG and MFS. To our best of knowledge, the comorbidity of MG and MFS is even rarer with only four reported cases. Some hypotheses are discussed; however, the underlying mechanisms remain to be elucidated in the future.

\section{Acknowledgments}

This study was funded by National Natural Science Foundation of China (81301016) and Beijing Municipal Administration of Hospitals' Youth Programme (QML20150303).

\section{Conflict of interest}

The authors declare no conflict of interest.

\section{References}

1. Mitsui T, Kuroda Y, Ueno S, et al. FK506 attenuates thymic output in patients with myasthenia gravis. Arch Med Sci 2013; 9: 1090-6.

2. Gilhus NE, Verschuuren JJ. Myasthenia gravis: subgroup classification and therapeutic strategies. Lancet Neurol 2015; 14: 1023-36.

3. Willison HJ, Jacobs BC, van Doorn PA. Guillain-Barre syndrome. Lancet 2016; 388: 717-27.

4. Ansar V, Valadi N. Guillain-Barre syndrome. Prim Care 2015; 42: 189-93.

5. Aranyi Z, Kovacs T, Sipos I, et al. Miller Fisher syndrome: brief overview and update with a focus on electrophysiological findings. Eur J Neurol 2012; 19: 15-20, e1-3.
6. Mak W, Chan KH, Ho SL. A case of ocular myasthenia gravis and Miller-Fisher syndrome. Hosp Med 2005; 66 116-7.

7. Silverstein MP, Zimnowodzki S, Rucker JC. Neuromuscular junction dysfunction in Miller Fisher syndrome. Semin Ophthalmol 2008; 23: 211-3.

8. Lau KK, Goh KJ, Lee HC, et al. The co-occurrence of serologically proven myasthenia gravis and Miller Fisher/Guillain Barre overlap syndrome: a case report. J Neurol Sci 2009; 276: 187-8.

9. Tanaka Y, Satomi K. Overlap of myasthenia gravis and Miller Fisher Syndrome. Intern Med 2016; 55: 1917-8.

10. Casetta I, Groppo E, De Gennaro R, et al. Myasthenia gravis: a changing pattern of incidence. J Neurol 2010; 257: 2015-9.

11. Bullens RW, O'Hanlon GM, Wagner E, et al. Complex gangliosides at the neuromuscular junction are membrane receptors for autoantibodies and botulinum neurotoxin but redundant for normal synaptic function. J Neurosci 2002; 22: 6876-84.

12. Krampfl K, Mohammadi B, Buchwald B, et al. IgG from patients with Guillain-Barre syndrome interact with nicotinic acetylcholine receptor channels. Muscle Nerve 2003; 27: 435-41.

13. O'Hanlon GM, Bullens RW, Plomp JJ, et al. Complex gangliosides as autoantibody targets at the neuromuscular junction in Miller Fisher syndrome: a current perspective. Neurochem Res 2002; 27: 697-709.

14. Romi F, Gilhus NE, Aarli JA. Myasthenia gravis: clinical, immunological, and therapeutic advances. Acta Neurol Scand 2005; 111: 134-41.

15. Carlander B, Touchon J, Georgesco M, et al. Myasthenia gravis and recurrent Guillain-Barre syndrome. Neurology 1991; 41: 1848.

16. Kimura K, Nezu A, Kimura S, et al. A case of myasthenia gravis in childhood associated with chronic inflammatory demyelinating polyradiculoneuropathy. Neuropediatrics 1998; 29: 108-12.

17. Wang JY, Pan J, Luo BY, et al. Temporal coincidence of myasthenia gravis and Guillain Barre syndrome associated with Hashimoto thyroiditis. Neurol Sci 2011; 32: 515-7. 